\title{
Solidification of helium, at room temperature under high pressure $\left({ }^{*}\right)$
}

\author{
J.-P. Pinceaux $\left({ }^{\dagger}\right)$, J.-P. Maury and J.-M. Besson $\left(^{\dagger}\right)$ \\ Laboratoire de Physique des Solides (**) and ( $\dagger$ ) Département de Hautes Pressions, \\ Université Pierre-et-Marie-Curie, 4, place Jussieu, 75230 Paris Cedex 05, France
}

(Reçu le 25 avril 1979, accepté le 11 mai 1979)

\begin{abstract}
Résumé. - La cristallisation de l'hélium ${ }^{4} \mathrm{He}$ a été observée à $297 \mathrm{~K}$ et $115 \pm 2$ kilobars. Cette valeur est proche de celle donnée par l'équation de Simon, extrapolée de mesures à 5 kilobars. La cellule diamant utilisée est d'une conception nouvelle qui permet l'étude de tous les gaz inertes entre 4 et $500 \mathrm{~K}$ jusqu'à 1 mégabar.
\end{abstract}

\begin{abstract}
Melting of ${ }^{4} \mathrm{He}$ has been observed at $297 \mathrm{~K}$ and $115 \pm 2$ kilobars. This value is close to that derived from Simon's equation, extrapolated from 5 kilobar data. The diamond cell we used is a new design and allows inert gases to be studied between 4 and $500 \mathrm{~K}$ up to 1 megabar.
\end{abstract}

1. Introduction. - Melting curves at high pressures and temperatures, and, in general thermodynamic parameters of matter at high densities are of interest in numerous fields of research, especially as diamond anvil techniques now reach into the megabar range $(100 \mathrm{GPa})$ or more.

Helium is of particular interest for the study of melting parameters since its critical point is the lowest of all elements. Its melting curve has only been measured up to 14 kilobars at liquid $\mathrm{N}_{2}$ temperature, and, in this range, is quite well represented by Simon's equation. Over 40 years ago, L. D. Landau showed the impossibility of a terminal critical point on a melting curve, but switching of the transition from first to second order [2] should not be completely ruled out. There is also a possibility of a temperature maximum [3] on the melting curve which would indicate the approach of a triple point and of a solidsolid transition [4], although it has been proved that both of these conjectures do not hold for elements in the argon and sodium classes [5].

Outside the field of fundamental thermodynamics, there is evident need for better knowledge of the behaviour of gases (fluids) at very high densities, particularly hydrogen (deuterium, tritium), helium and their mixtures. In the field of very high pressure research in the diamond cell, helium has been thought

(*) La version française de cet article paraîtra dans le $n^{\circ} 16$ du 2 mai 1979 des C.R.A.S.

(**) Associé au C.N.R.S. to be a convenient pressure transmitting medium : because of its presumably low rigidity, strain gradients in solid $\mathrm{He}$ would be lower than in the ethanolmethanol glasses now used above 100 kilobars.

The data reported here have been obtained by a technique derived from the classical clamp-type diamond anvil cells, using the shift of the ruby luminescence as a pressure gauge [6]. Diamond cells have been shown to generate pressures above 1 megabar [7] on solid samples. Recently [8], crystallization of $\mathbf{H}_{2}$ was observed at 57 kilobars and $298 \mathrm{~K}$ in a device with cryogenic filling, to enclose a sample with high enough initial density. We reached the same result with a different method by filling the sample volume with helium under 2000 bar-pressure, at room temperature. We could thus observe and measure the fluid-solid equilibrium in helium at room temperature.

2. Experimental. - Diamond anvils and seats are of the conventional form [6, 9]. An Inconel 750 gasket was used. The thrust on the diamonds is exerted by an annular piston with a $1 \mathrm{~cm}^{2}$ effective area, backed with a few hundred bars gas pressure. For filling, the cell is enclosed in a low pressure bomb which is then brought to 2000 bars pressure. This ensures sufficient density for the gas under study, which fills the sample volume between the anvils. The piston which supports the sliding diamond is then put under 250 bars gas overpressure ( 2250 bars in our example). This brings the diamonds in contact with the gasket and isolates the sample volume. In our case, helium is then under a pressure near 100 kilobar. Pressures 
in the bomb and on the piston are then gradually decreased, preserving the same differential pressure, until the former is zero. The cell is then extracted from the bomb and contains a significant mass of helium $\left(\sim 10^{-7}\right.$ grams). The internal pressure can be varied by varying the low pressure backing the piston, which remains attached, by a capillary, to the low-pressure generator through the whole process. Details of this setup as well as experimental procedures will be fully described in a later paper [10].

In our experiments, we used U-grade helium with impurity content below $20 \mathrm{ppm}$. Crystallization comes about rapidly upon compression and the value of pressure is not well defined. The equilibrium point was therefore determined at the coexistence of the solid and liquid phases, during slow decompression. Direct microscopic observation showed the melting of large $(50 \times 30 \mu)$ crystals during $15 \mathrm{~min}$. Those conditions can be considered as isothermal in view of the thickness $(\sim 20 \mu)$ of the cell, and thermal conductivity of the anvils.

The equilibrium pressure is $115 \pm 2$ kilobars at $297 \pm 0.4 \mathrm{~K}$.

The refractive index difference between the two phases is quite weak which is not unexpected for helium. Crystals were observable only under oblique illumination. No anisotropy is apparent under crossed polarizers.

3. Conclusions. - The index difference of the two phases which renders the crystals visible clearly points to a difference in density between the solid and the fluid : the transition is thus of the first order up to 115 kilobars.

Room temperature $(297 \mathrm{~K})$, is over 50 times the critical temperature for ${ }^{4} \mathrm{He}$. Comparison with Simon's equation with the parameters quoted in ref. [12] gives a predicted value of 115 kilobars, which fits well with our value of $115 \pm 2$ kilobars. The figure shows a comparison of this equation with existing data. Thus, the extrapolation of this type of melting curve can be safely used above 100 kilobars for helium and, $a$ fortiori, for other elements verifying Simon's equation whose critical temperature is higher.

There is no evidence for a solid-solid phase transition in helium up to 120 kilobars : the melting curve shows no inflexion point that could be linked to proximity of a triple point. No index anisotropy in the

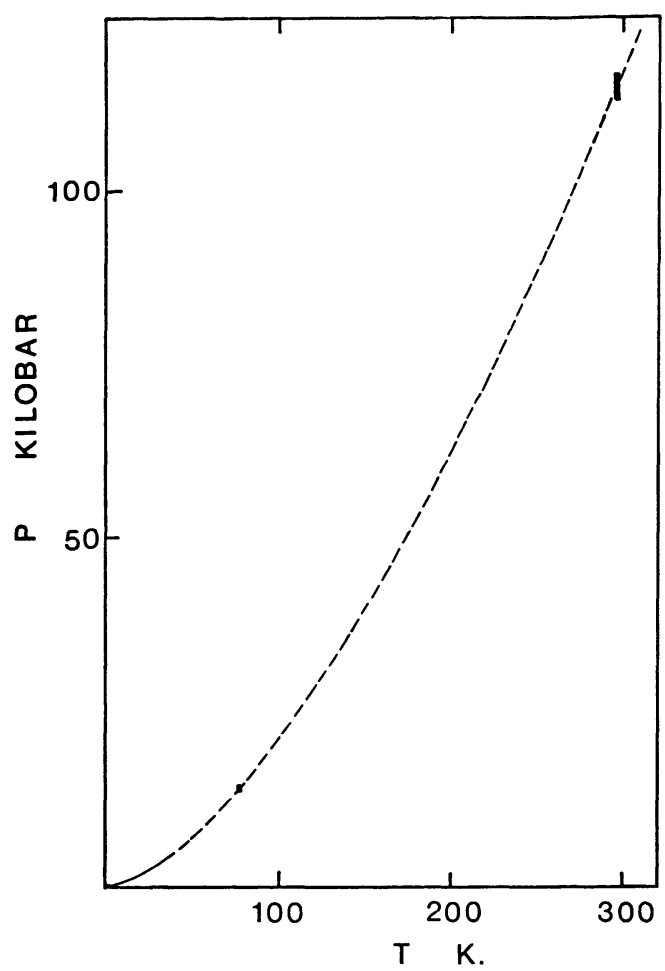

Fig. 1. - Melting curve of helium. The dashed curve is calculated following Simon's equation [12]

$$
P=16.235\left[(T / 0.992)^{1.5554}-1\right] .
$$

The full line up to 5 kilobars represents the measurements of Holland et al., and Robinson (Ref. [12]). At $77 \mathrm{~K}$ : Langer's measurements. At $297 \mathrm{~K}$ : our measurements.

solid could be observed, which points to a situation where helium has retained its face-centred cubic low-pressure structure.

No broadening of the ruby luminescence has been observed, in the solid phase. This confirms the expected plasticity of solid helium at high pressures. Moreover, the refractive index being low, grain boundaries are weak and transparency of the medium is good : solid helium seems to be a convenient pressure-transmitting medium for optical measurements above 100 kilobars, especially in view of its chemical inertness in comparison with classical ethanol-methanol mixtures [9].

Finally, concerning high pressure technology, our setup is applicable to the study of all fluids which are chemically compatible with the cell materials, in the megabar range and between 4 and $500 \mathrm{~K}$.

\section{References}

[1] Landau, L. D., Collected papers (Pergamon press, Oxford) 1965

[2] Lennard Jones, J. E., Devonshire, A. F., Proc. R. Soc. A 170 (1939) 464

[3] Tammann, G., Moritz, G., Z. Anorg. Allgem. Chem. 218 (1934) 60 .

[4] Stishov, S. M., Sov. Phys.-USPEKHI 11 (1969) 816.

[5] Stishov, S. M., Sov. Phys.-USPEKHI 17 (1974) 525.

[6] Piermarini, G. J., Block, S., Rev. Sci. Instrum. 46 (1975) 973.
[7] MaO, H. K., Bell, P. M., Science 200 (1978) 1145.

[8] MaO, H. K., Bell, P. M., Science 203 (1979) 1004.

[9] Besson, J. M., Pinceaux, J. P., Rev. Sci. Instrum. 50 (1979) 79.

[10] Pinceaux, J. P., In preparation for submission to Rev. Sci. Instrum.

[11] Simon, F., Ruhemann, M., Edwards, W., Z. Phys. Chem. B 6 (1929) 62.

[12] Langer, D. W. J., J. Phys. Chem. Solids 1 (1961) 123. 